\title{
Pessoas que vivem com HIV/AIDS em Florianópolis/SC, Brasil: ocupação e status socioeconômico ocupacional (1986-2006)
}

Persons living with HIV/AIDS in Florianópolis/SC, Brazil: occupation and occupational socioeconomic status (1986-2006)

Personas que viven con HIV/SIDA en Florianópolis-SC, Brasil: ocupación y status socioeconómico ocupacional (1986-2006)

Janelice de Azevedo Neves Bastiani ${ }^{1}$, Maria Itayra Coelho de Souza Padilha' ${ }^{2}$ Mariana Vieira ${ }^{3}$, Isabel Cristina Alves Maliska ${ }^{4}$, Ana Rosete Camargo Rodrigues Maia ${ }^{5}$

\section{RESUMO}

Este estudo teve como objetivo analisar a distribuição das pessoas adultas que vivem com a Síndrome da Imunodeficiência Humana Adquirida (AIDS) em Florianópolis/SC, entre 1986 e 2006. Estudo histórico de 3.209 casos de AIDS, com dados coletados no Setor de Vigilância Epidemiológica da Secretaria Municipal de Saúde, entre março e abril de 2010. A análise dos dados aborda ocupação no mercado de trabalho e sexo; condição socioeconômica dos casos com idade entre 20 e 49 anos; categoria de exposição e sexo. A AIDS afeta subgrupos e vulneráveis potenciais. Mulheres ocupam a Categoria Não Ocupada nos Afazeres Domésticos e homens ocupam a Categoria Não Ocupado como Estudantes e Aposentados/Pensionistas. Quanto ao Status Socioeconômico Ocupacional, as mulheres se encontram entre o $4^{\circ}$ e $5^{\circ}$, e os homens entre o $1^{\circ}$ e $5^{\circ}$. Os resultados sugerem que as ações de prevenção ao HIV/AIDS devem ser planejadas para atendimento dos subgrupos com vulnerabilidade potencial.

Descritores: Síndrome da Imunodeficiência Adquirida; Ocupação; Fatores Socioeconômicos.

\section{ABSTRACT}

The objective of this study was to analyze the distribution of adult persons living with Acquired Immunodeficiency Syndrome (AIDS) in Florianópolis/SC between 1986 and 2006. This is a historical study of 3,209 individuals with AIDS, utilizing data collected from the Epidemiology Surveillance Sector of the Municipal Health Department between March and April of 2010. Data analysis addresses the type of occupation in the labor market; socioeconomic status of individuals aged 20 to 49 years; category of exposure and gender. AIDS affects subgroups and potentially vulnerable individuals. Women prevail in the Unemployed category as housewives and men occupy the Unemployed category as students and retirees/pensioners. Regarding occupational socioeconomic status, women were ranked between the 4th and 5th, and men between the 1st and 5th. The results suggest that HIV/AIDS prevention activities should be planned to address the subgroups that are potentially vulnerable.

Descriptors: Acquired Immunodeficiency Syndrome; Occupancy; Socioeconomic Factors.

\section{RESUMEN}

Se objetivó analizar la distribución de personas adultas con Síndrome de Inmunodeficiencia Adquirida (SIDA) en Florianópolis-SC, de 1986 a 2006. Estudio histórico de 2306 casos de SIDA. Datos recolectados en sector de Vigilancia Epidemiológica de la Secretaría Municipal de Salud de Florianópolis-SC, entre marzo y abril de 2010. El análisis de datos aborda ocupación en el mercado de trabajo y sexo; condición socioeconómica de los casos con edades entre 20 y 49 años; categoría de exposición y sexo. El SIDA afecta a subgrupos y vulnerables potenciales. Las mujeres se ubican en la Categoría No Ocupada de Quehaceres Domésticos, los hombres en la Categoría No Ocupada como Estudiantes y Jubilados/Pensionados. Respecto al Status Socioeconómico Ocupacional, las mujeres se encuentran entre la $4^{\mathrm{a}}$ y $5^{\mathrm{a}}$, y los hombre entre la $1^{\mathrm{a}}$ y $5^{\mathrm{a}}$. Los resultados sugieren que las acciones preventivas para HIV/SIDA deben planificarse para atender a los subgrupos de vulnerabilidad potencial.

Descriptores: Síndrome de Inmunodeficiencia Adquirida; Ocupación; Factores Socioeconómicos.

\footnotetext{
1 Enfermeira, Mestre em Enfermagem. Enfermeira da Secretaria Municipal de Saúde de Florianópolis. Florianópolis, SC, Brasil. E-mail: bastiani@ibest.com.br.

${ }^{2}$ Enfermeira, Doutora em Enfermagem. Professora Associada da Universidade Federal de Santa Catarina (UFSC). Florianópolis, SC, Brasil. E-mail: padilha@ccs.ufsc.br.

${ }^{3}$ Enfermeira, Mestre em Enfermagem. Discente do Programa de Pós-Graduação em Enfermagem da UFSC (PEN/UFSC), nível Doutorado. Florianópolis,

SC, Brasil. E-mail: nanyufsc@ibest.com.br.

${ }^{4}$ Enfermeira, Mestre em Enfermagem. Discente do PEN/UFSC, nível Doutorado. Florianópolis, SC, Brasil. E-mail: isabel.alves07@yahoo.com.br.

${ }^{5}$ Enfermeira, Doutora em Enfermagem. Professora Adjunta da UFSC. Florianópolis, SC, Brasil. E-mail: maia@nfr.ufsc.br.
} 


\section{INTRODUÇÃO}

Os primeiros casos da Síndrome da Imunodeficiência Adquirida (AIDS) foram identificados no mundo na década de 80 , entre homens, hemofílicos, homossexuais (HSH) e/ou usuários de drogas (UDI), com boas condições socioeconômicas; pessoas que partilhavam destas características foram consideradas como pertencentes a grupos em risco de contrair o Vírus da Imunodeficiência Humana (HIV) e desenvolver a AIDS. Neste contexto, pessoas que não pertenciam a estes grupos de risco, passaram a perceber-se como imunes ao HIV/AIDS. Esta concepção tem sido desconstruída no decorrer dos anos, visto que todos podem infectar-se pelo HIV, ao exporemse aos modos de transmissão sem proteção adequada ${ }^{(1)}$.

No Brasil, entre 1980 a junho de 2011, foram notificados 608.230 casos de AIDS, sendo 397.662 $(65,4 \%)$ no sexo masculino e $210.538(34,6 \%)$ no sexo feminino, com a razão entre os sexos diminuindo sistematicamente a partir de 1998. Atualmente, a AIDS no Brasil se concentra em subgrupos populacionais com idade entre 20 a 59 anos, com um a 11 anos de estudo e razão entre os sexos de 1,7 homens para uma mulher com AIDS, segundo dados do ano de 2011(2).

O Programa das Nações Unidas para o Desenvolvimento (PNUD) da Organização das Nações Unidas (ONU) é uma rede composta por 166 países com objetivo de combater a pobreza, proporcionar acesso a recursos técnicos, humanos e financeiros aos países membros do PNUD. Para verificar o desenvolvimento humano nos países, o PNUD orienta considerar os aspectos econômicos, sociais, culturais e políticos na averiguação da qualidade de vida da população(3).

O Brasil segue as orientações do PNUD, ao aferir a qualidade de vida da população através do Instituto Brasileiro de Geografia e Estatística (IBGE), que quantifica os indicadores econômicos, sociais, culturais e políticos, que caracterizam a qualidade de vida da população brasileira. Na década de 80 , as famílias brasileiras tinham como características serem chefiadas por mulheres, $67,5 \%$ das famílias viviam nas cidades, e $12,5 \%$ das mulheres da área rural não eram alfabetizadas. Na década de 90 , a taxa de crescimento da população foi de $1,3 \%$ e $78,4 \%$ das famílias residiam nas cidades. Fatores como, a recessão econômica entre os anos de 90 a 92, a abertura comercial, a estabilização econômica e as privatizações, passaram a alterar a ocupação laboral, o rendimento e a educação da população brasileira ${ }^{(4)}$.

A variação na qualidade de vida da população brasileira criou diversos estratos sociais, nos quais se inserem os subgrupos vulneráveis ao HIV/AIDS, cada vez mais associados ao processo de pauperização. Este fato tem contribuído para situações de abandono do tratamento, agravamento do adoecimento e consequentemente, aumento dos gastos no tratamento das intercorrências das pessoas que vivem com 0 HIV/AIDS ${ }^{(5)}$.

A taxa de prevalência do HIV em subgrupos no Brasil utiliza o referencial de cálculo elaborado pela Organização Mundial da Saúde (OMS) e aperfeiçoado pela Joint United Nations Programme on HIV/AIDS (UNAIDS). A taxa de prevalência fornece elementos para o planejamento e estruturação das ações de promoção da saúde e prevenção da AIDS e auxilia na elaboração das políticas nacionais as Doenças Sexualmente Transmissiveis (DST) e a AIDS(6).

Pesquisadores reforçam a importância de se conhecer os fatores envolvidos na transmissão do HIV, assim como a dinâmica social da transmissibilidade, pois se acredita na vulnerabilidade potencial de alguns membros dos subgrupos. A vulnerabilidade potencial leva os expostos ao HIV/AIDS ao agravamento das condições de vida e de saúde, ao se conviver com mais esta condição(5-6). Neste sentido, um melhor entendimento da dinâmica social da AIDS requer o aprimoramento das informações sociais disponíveis, visto que as medidas de prevenção e controle da epidemia não atingem homogeneamente a população(7).

No Brasil, no ano de 2010, a faixa etária que exibiu maior taxa de incidência no país foi de 35 a 39 anos de idade (38,1 casos/100.000 hab.). Ainda com relação às faixas etárias, entre 1998 e 2010, observou-se um aumento da taxa de incidência de casos de AIDS nas faixas etárias de 05 a 12, de 50 a 59, e de 60 anos e mais. Ainda este ano, as duas capitais federativas do Brasil com maior número de casos de AIDS por 100.000 habitantes foram da Região Sul, em primeiro lugar Porto Alegre/RS com 99,8, em segundo lugar Florianópolis/SC com $57,9^{(8)}$.

O Boletim Epidemiológico da Divisão de Epidemiologia de Santa Catarina revela que entre 1984 a 2010, foram notificados 4.035 casos de AIDS em 
Florianópolis/SC, ultrapassando outras 14 grandes cidades do estado(8).

Florianópolis/SC, no final da década de 80 experimentou um grande crescimento econômico, turístico e cultural, incentivado pela divulgação na mídia nacional da qualidade de vida na cidade, ocasionando um processo migratório interno e externo. Pessoas/famílias oriundas de várias cidades de Santa Catarina e de outros Estados das Regiões Sul e Sudeste, migraram para Florianópolis/SC em busca de emprego e de qualidade de vida. Entretanto, nas Áreas de Interesse Social (AIS) se fixaram pessoas/famílias com condição social, econômica, política e cultural precária, propensas a vulnerabilidade econômica e social, bem como a condições que antecedem e favorecem à exposição ao HIV e adoecimento da $\operatorname{AIDS}^{(5,8)}$.

Partindo deste contexto, buscamos analisar a dinâmica da AIDS em adultos residentes em Florianópolis/SC, no período de 1986 a 2006. O marco inicial do estudo é o ano de 1986, que configura a notificação do primeiro caso de AIDS em Florianópolis/SC no Sistema Nacional de Notificação de Agravos (SINAN). O marco final é o ano de 2006, em que o atendimento das DST/AIDS pelo serviço municipal de saúde é desmembrado, estruturando-se um ambulatório de DST/AIDS na área insular e outro na área continental de Florianópolis/SC.

O presente estudo, portanto, teve o objetivo de analisar a distribuição das pessoas adultas que vivem com HIV/AIDS em Florianópolis/SC, entre 1986 a 2006, em relação à categoria de ocupação e status socioeconômico ocupacional, desvelando a dinâmica social da AIDS em subgrupos vulneráveis, na perspectiva de contribuir no planejamento, avaliação e reorganização das políticas para as DST/AIDS no Brasil.

\section{METODOLOGIA}

Estudo histórico, descritivo, que utiliza dados das pessoas que vivem com HIV/AIDS em Florianópolis/SC, notificadas no Sistema Nacional de Agravos de Notificação (SINAN - AIDS) e Sistema de Informação de Mortalidade (SIM), entre os anos de 1986 a 2006. Os dados foram coletados no Setor de Vigilância Epidemiológica da Secretaria Municipal de Saúde de Florianópolis, nos meses de março e abril de 2010.
Na primeira parte do estudo, as ocupações das pessoas que vivem com HIV/AIDS em Florianópolis/SC, foram estabelecidas conforme a Classificação Вrasileira de Ocupações (CBO) do Ministério do Trabalho e categorizadas a partir da proposta do estudo de Fonseca ${ }^{(9)}$

As pessoas que vivem com HIV/AIDS em Florianópolis/SC foram agrupadas em dois grupos: "ocupados" e "não ocupados", sendo considerados como "ocupados" aquelas com qualquer ocupação contida na "Relação de Códigos de Ocupação" definidos pelo Instituto Brasileiro de Geografia e Estatística (IBGE) de 1998. Рara a categorização dos "ocupados", utilizou-se a classificação de ocupações composta originalmente por 16 categorias ocupacionais, definidas a partir da agregação dos códigos de ocupação do IBGE de 1998, sendo nove do estrato "não-manual" e sete do estrato "manual"; os sem ocupação foram categorizados em "não ocupados" no estrato quatro.

Incluiu-se na categoria profissional "Não - Manuais" os Autônomos e os Trabalhadores Sem Ocupação Definida; na categoria ocupacional "Não Ocupado" incluímos os Presidiários, pois Florianópolis/SC conta com uma unidade prisional de grande porte.

Cada variável denominada ocupação pelo SINAN/AIDS foi agregada, conforme a categoria ocupacional descrita, recebendo codificação de um a 20 e com variáveis coletadas no SINAN/AIDS (ano de diagnóstico, sexo e categoria de exposição). Após, os dados foram cadastrados em planilha do Programa Excel e analisadas com auxílio do programa Epi Info 3.5.1.

Na segunda parte do estudo, optou-se em proceder a avaliação do Status Socioeconômico Ocupacional (ESO) das PVHA com idade entre 20 a 49 anos, de ambos os sexos, no período 1986 a 2006. As categorias de exposição selecionadas foram: homossexual, bissexual (ambas exclusivas para o sexo masculino), heterossexual, UDI e ignorado(2).

As categorias ocupacionais foram codificadas de um a cinco, procedeu-se a categorização por sexo, categoria de exposição, conforme indicadores do ESO. Nessa etapa retirou-se a categoria ocupacional "Não - Ocupados", por não expressarem atividade laboral e remunerativa ${ }^{(9)}$.

O estudo foi aprovado pelo Comitê de Ética em Pesquisa da Universidade Federal de Santa Catarina em 
05 de novembro de 2009, através do processo № $331 / 2009$.

\section{RESULTADOS}

Os dados analisados correspondem as 3.209 notificações de casos de AIDS em Florianópolis/SC entre 1986 a 2006, sendo respectivamente $2.160(67,4 \%)$ notificações do sexo masculino e $1.046(32,6 \%)$ notificações do sexo feminino.

Em relação à distribuição das Categorias Ocupacionais das pessoas que vivem com HIV/AIDS em Florianópolis/SC, se observa as diferentes atividades laborais exercidas, conforme os dados da Tabela 1.

Tabela 1: Distribuição das PVHA, segundo inserção no mercado de trabalho, sexo masculino e ocupação no município de Florianópolis, SC, Brasil, 1986 a 2006.

\begin{tabular}{|c|c|c|c|c|}
\hline \multicolumn{5}{|c|}{ PERÍODO } \\
\hline \multirow{2}{*}{ CATEGORIAS } & \multicolumn{2}{|c|}{$1986-1995$} & \multicolumn{2}{|c|}{$1996-2006$} \\
\hline & $\mathbf{N}$ & $\%$ & $\mathbf{N}$ & $\%$ \\
\hline IGNORADO & 14 & 1,7 & 57 & 4,3 \\
\hline \multicolumn{5}{|l|}{ NÃO - MANUAL } \\
\hline Proprietários Rurais & 0 & 0 & 0 & 0 \\
\hline Profissionais Liberais & 18 & 2,2 & 14 & 1,0 \\
\hline Outros Profissionais Liberais & 83 & 10,1 & 57 & 4,3 \\
\hline Empregados & 1 & 0,1 & 0 & 0 \\
\hline Empresários por Conta Própria & 34 & 4,1 & 37 & 2,8 \\
\hline Dirigentes & 1 & 0,1 & 3 & 0,2 \\
\hline Ocupações Técnicas e Artísticas & 11 & 1,3 & 28 & 2,1 \\
\hline Ocupações não Manuais de Rotina & 1 & 0,1 & 4 & 0,3 \\
\hline Supervisores do Trabalho Manual & 2 & 0,2 & 4 & 0,3 \\
\hline \multicolumn{5}{|l|}{ MANUAL } \\
\hline Trabalhadores Rurais & 5 & 0,6 & 4 & 0,3 \\
\hline Trabalhadores da Indústria Moderna & 20 & 2,4 & 37 & 2,8 \\
\hline Trabalhadores da Indústria Tradicional & 164 & 19,9 & 291 & 21,8 \\
\hline Trabalhadores do Comércio Ambulante & 5 & 0,6 & 11 & 0,8 \\
\hline Trabalhadores nos Serviços Pessoais & 287 & 34,8 & 396 & 29,7 \\
\hline Trabalhadores nos Serviços Gerais & 69 & 8,3 & 94 & 7,0 \\
\hline Trabalhadores nos Serviços Domésticos & 0 & 0 & 0 & 0 \\
\hline Autônomas & 6 & 0,7 & 66 & 4,9 \\
\hline Trabalhadores em Classificação Definida & 86 & 10,3 & 157 & 11,7 \\
\hline \multicolumn{5}{|l|}{ NÃO OCUPADOS } \\
\hline Desempregados & 0 & 0 & 1 & 0,1 \\
\hline Estudantes & 13 & 1,6 & 32 & 2,4 \\
\hline Afazeres Domésticos & 1 & 0,1 & 3 & 0,2 \\
\hline Aposentadas e/ou Pensionistas & 3 & 0,4 & 25 & 1,9 \\
\hline Presidiários & 3 & 0,4 & 15 & 1,1 \\
\hline TOTAL & 827 & 100 & 1336 & 100 \\
\hline
\end{tabular}

Fonte: SINAN-AIDS. Março e Abril/2010.

No sexo masculino, na primeira década (1986-1995), a Categoria Ocupacional "Não Manual” destaca a atuação de 'Outros Profissionais Liberais e Empresários por Conta Própria', perfazendo 14,2\% dos homens que vivem com HIV/AIDS em Florianópolis/SC. Na segunda década (1996-2006), ocorre redução/elevação discreta em praticamente todas as categorias de ocupação, se destacando a Categoria 'Ocupações Técnicas e Artísticas', com acréscimo de 0,8\%; e queda para 5,8\% da ocupação 'Outros Profissionais Liberais' e 'Empresários por Conta Própria' entre os homens que vivem com HIV/AIDS.

Na primeira década (1986-1995), na Categoria Ocupacional "Manual", se destacam significativamente 
os 'Trabalhadores nos Serviços Pessoais' e 'Trabalhadores da Indústria Tradicional', totalizando $54,7 \%$ dos homens que vivem com HIV/AIDS em Florianópolis/SC. Na segunda década (1996-2006), se mantiveram as ocupações de 'Trabalhadores nos Serviços Pessoais' e 'Trabalhadores da Indústria Tradicional', aferindo $51,5 \%$ dos homens que vivem com HIV/AIDS em Florianópolis/SC. Apesar da redução de $3,2 \%$ nestas ocupações, permanece presente a vulnerabilidade potencial destas categorias. O número de homens distribuídos na categoria "Manual" obteve incremento percentual de 3,2\% nas ocupações laborais da categoria.
Na primeira década (1986-1995), na Categoria Ocupacional "Não - Ocupado" a categoria 'Estudantes', 'Aposentados e/ou Pensionista' e 'Presidiários', os resultados mais significativos foram entre os Estudantes com $1,6 \%$ dos homens que vivem com HIV/AIDS em Florianópolis/SC. Na segunda década (1996 a 2006), se observa entre os 'Estudantes' acréscimo de 0,8\%, com incremento de 1,5\% de 'Aposentados e/ou Pensionista' entre os homens que vivem com HIV/AIDS em Florianópolis/SC.

A distribuição dos dados nas Categorias Ocupacionais, no sexo feminino, entre as décadas de 1986 a 1995 e 1996 a 2006, estão distribuídas conforme a Tabela 2.

Tabela 2: Distribuição das PVHA, segundo inserção no mercado de trabalho, sexo feminino e ocupação no município de Florianópolis, SC, Brasil, 1986 a 2006.

\begin{tabular}{|c|c|c|c|c|}
\hline \multicolumn{5}{|c|}{ PERÍODO } \\
\hline \multirow{2}{*}{ CATEGORIAS } & \multicolumn{2}{|c|}{$1986-1995$} & \multicolumn{2}{|c|}{$1996-2006$} \\
\hline & $\mathbf{N}$ & $\%$ & $\mathbf{N}$ & $\%$ \\
\hline IGNORADO & 2 & 0,7 & 37 & 4,8 \\
\hline \multicolumn{5}{|l|}{ NÃO - MANUAL } \\
\hline Proprietárias Rurais & 0 & 0 & 0 & 0 \\
\hline Profissionais Liberais & 3 & 1,1 & 2 & 5,1 \\
\hline Outras Profissionais Liberais & 13 & 4,7 & 20 & 7,7 \\
\hline Empregadas & 0 & 0,0 & 0 & 0 \\
\hline Empresárias por Conta Própria & 5 & 1,8 & 17 & 9,9 \\
\hline Dirigentes & 0 & 0,0 & 0 & 9,9 \\
\hline Ocupações Técnicas e Artísticas & 1 & 0,4 & 5 & 10,5 \\
\hline Ocupações não Manuais de Rotina & 3 & 1,1 & 5 & 11,2 \\
\hline Supervisoras do Trabalho Manual & 0 & 0 & 1 & 11,3 \\
\hline \multicolumn{5}{|l|}{ MANUAL } \\
\hline Trabalhadoras Rurais & 0 & 0 & 0 & 0 \\
\hline Trabalhadoras da Indústria Moderna & 2 & 0,7 & 0 & 0 \\
\hline Trabalhadoras da Indústria Tradicional & 14 & 5,1 & 27 & 3,5 \\
\hline Trabalhadoras do Comércio Ambulante & 1 & 0,4 & 7 & 0,9 \\
\hline Trabalhadoras nos Serviços Pessoais & 77 & 27,7 & 134 & 17,4 \\
\hline Trabalhadoras nos Serviços Gerais & 18 & 6,5 & 95 & 12,4 \\
\hline Trabalhadoras nos Serviços Domésticos & 0 & 0 & 1 & 0,1 \\
\hline Autônomas & 1 & 0,4 & 16 & 2,0 \\
\hline Trabalhadoras em Classificação Definida & 16 & 5,7 & 63 & 8,2 \\
\hline \multicolumn{5}{|l|}{ NÃO OCUPADAS } \\
\hline Desempregadas & 0 & 0 & 0 & 0 \\
\hline Estudantes & 11 & 4,0 & 23 & 3,0 \\
\hline Afazeres Domésticos & 108 & 39,0 & 300 & 39,1 \\
\hline Aposentadas e/ou Pensionistas & 2 & 0,7 & 13 & 1,7 \\
\hline Presidiárias & 0 & 0 & 3 & 0,4 \\
\hline TOTAL & 277 & 100 & 769 & 100 \\
\hline
\end{tabular}

Fonte: SINAN-AIDS. Março e Abril, 2010. 
Na primeira década (1986-1995), na Categoria de Ocupação "Não - Manual", 12,4\% das mulheres que vivem com HIV/AIDS em Florianópolis/SC atuavam como 'Outras Profissionais Liberais' e 'Empresárias por Conta Própria'. Na segunda década (1996-2006), na Categoria de Ocupação "Não - Manual", as 'Ocupações Técnicas e Artísticas', 'Ocupações não Manuais de Rotina' e 'Supervisoras do Trabalho Manual' concentraram 33\% das mulheres que vivem com HIV/AIDS em Florianópolis/SC.

Na primeira década (1986-1995), na Categoria Ocupacional "Manual", 34,2\% das mulheres que vivem com HIV/AIDS em Florianópolis/SC, atuaram como 'Trabalhadoras nos Serviços Pessoais' e 'Trabalhadoras sem Classificação Definida'. Na segunda década (19962006), 29,8\% das mulheres que vivem com HIV/AIDS em Florianópolis/SC exerceram ocupações como 'Trabalhadoras nos Serviços Pessoais' e 'Trabalhadoras nos Serviços Gerais'. Ocupações como 'Trabalhadoras nos Serviços Pessoais' e 'Trabalhadoras nos Serviços Gerais' apresentaram diminuição de 4,4\% como atividade laboral exercida pelas mulheres que vivem com HIV/AIDS em Florianópolis/SC, entretanto em outras ocupações ocorreu elevação dos percentuais do exercício profissional.

Na primeira década (1986-1995), na Categoria Ocupacional "Não - Ocupada", 39,0\% das mulheres que vivem com HIV/AIDS em Florianópolis/SC exerceram atividades de 'Afazeres Domésticos'. Na segunda década (1996-2006), 39,1\% das mulheres que vivem com HIV/AIDS em Florianópolis/SC, tiveram como atividade laboral os 'Afazeres Domésticos'. Nas duas décadas do estudo, observa-se mínima variação entre mulheres que vivem com HIV/AIDS exercendo atividades como os Afazeres Domésticos, destacando-se como subgrupo de vulnerabilidade potencial.

Dos dados analisados, sobre o Status Socioeconômico Ocupacional (ESO), das 1.565 pessoas com idade entre 20 e 49 anos que vivem com HIV/AIDS em Florianópolis/SC, estas estão distribuídas em $1.191(76,1 \%)$ do sexo masculino e $374(23,9 \%)$ do sexo feminino.

Tabela 3: Distribuição das PVHA, segundo categoria de exposição, sexo, em indivíduos entre 20 a 49 anos e ESO no município de Florianópolis, SC, Brasil, 1986 a 2006.

\begin{tabular}{|c|c|c|c|c|c|c|c|}
\hline \multirow{2}{*}{ CATEGORIA DE EXPOSIÇÃO } & \multirow{2}{*}{ PERÍODO } & \multirow{2}{*}{$\mathbf{N}$} & \multicolumn{5}{|c|}{ STATUS SOCIOECONÔMICO (ESO) } \\
\hline & & & 10 & $2^{\circ}$ & $3^{\circ}$ & $4^{\circ}$ & $5^{\circ}$ \\
\hline \multicolumn{8}{|c|}{ SEXO FEMININO } \\
\hline \multirow{2}{*}{ HETEROSSEXUAL } & $1986-1995$ & 93 & $15,0 \%$ & $3,2 \%$ & $2,2 \%$ & $60,2 \%$ & $19,4 \%$ \\
\hline & $1996-2006$ & 239 & $9,6 \%$ & $2,9 \%$ & $0,0 \%$ & $41,8 \%$ & $45,7 \%$ \\
\hline \multirow{2}{*}{ UDI } & $1986-1995$ & 24 & $12,5 \%$ & $0,0 \%$ & $0,0 \%$ & $75,0 \%$ & $12,5 \%$ \\
\hline & $1996-2006$ & 4 & $0,0 \%$ & $0,0 \%$ & $0,0 \%$ & $50,0 \%$ & $50,0 \%$ \\
\hline \multirow{2}{*}{ IGNORADO } & $1986-1995$ & 0 & $0,0 \%$ & $0,0 \%$ & $0,0 \%$ & $0,0 \%$ & $0,0 \%$ \\
\hline & $1996-2006$ & 14 & $7,1 \%$ & $7,1 \%$ & $0,0 \%$ & $42,9 \%$ & $42,9 \%$ \\
\hline \multicolumn{8}{|c|}{ SEXO MASCULINO } \\
\hline \multirow{2}{*}{ HOMOSSEXUAL } & $1986-1995$ & 217 & $1240,0 \%$ & $1,4 \%$ & $3,7 \%$ & $61,8 \%$ & $20,7 \%$ \\
\hline & $1996-2006$ & 112 & $260,0 \%$ & $0,0 \%$ & $3,6 \%$ & $58,0 \%$ & $35,8 \%$ \\
\hline \multirow{2}{*}{ BISSEXUAL } & $1986-1995$ & 42 & $2390,0 \%$ & $0,0 \%$ & $2,4 \%$ & $59,5 \%$ & $14,2 \%$ \\
\hline & $1996-2006$ & 65 & $920,0 \%$ & $1,8 \%$ & $1,5 \%$ & $66,2 \%$ & $21,6 \%$ \\
\hline \multirow{2}{*}{ HETEROSSEXUAL } & $1986-1995$ & 108 & $23,1 \%$ & $1,8 \%$ & $4,6 \%$ & $51,0 \%$ & $19,5 \%$ \\
\hline & $1996-2006$ & 258 & $7,0 \%$ & $4,2 \%$ & $5,0 \%$ & $54,7 \%$ & $29,1 \%$ \\
\hline \multirow{2}{*}{ UDI } & $1986-1995$ & 217 & $12,3 \%$ & $1,4 \%$ & $3,6 \%$ & $62,0 \%$ & $20,7 \%$ \\
\hline & $1996-2006$ & 112 & $2,7 \%$ & $0,0 \%$ & $3,6 \%$ & $58,0 \%$ & $35,7 \%$ \\
\hline \multirow{2}{*}{ IGNORADO } & $1986-1995$ & 2 & $50,0 \%$ & $0,0 \%$ & $50,0 \%$ & $0,0 \%$ & $0,0 \%$ \\
\hline & $1996-2006$ & 58 & $8,6 \%$ & $1,7 \%$ & $3,4 \%$ & $44,9 \%$ & $41,4 \%$ \\
\hline
\end{tabular}

Na primeira década (1986-1995), observa-se que entre o sexo feminino a infecção pelo HIV predominou nas categorias de exposição Heterossexual e UDI, e na relação categoria de exposição e Status Socioeconômico 
Ocupacional, estas mulheres localizam-se no $4^{\circ}$ ESO. Na segunda década (1996-2006), observa-se entre mulheres que vivem com HIV/AIDS em Florianópolis/SC, a categoria de exposição Heterossexual se encontra no $5^{\circ}$ ESO, enquanto que a categoria UDI e Ignorado prevaleceu no $4^{\circ}$ e $5^{\circ}$ ESO. O resultado sinaliza a vulnerabilidade potencial em subgrupos de mulheres com baixa/nenhuma escolaridade, que exercem atividades laborais de baixa remuneração.

Na primeira década (1986-1995), observa-se entre os homens que vivem com HIV/AIDS em Florianópolis/SC, a exposição nas categorias Homossexual, Bissexual, Heterossexual e UDI. Quando analisados os dados sobre o Status Socioeconômico Ocupacional, o sexo masculino se concentra no $4^{\circ}$ e $5^{\circ}$ ESO. Na segunda década (1996-2006), eleva-se em todas as categorias de exposição à concentração de homens no $5^{\circ}$ ESO. O resultado aponta o declínio do padrão socioeconômico no sexo masculino, com redução do alto padrão socioeconômico para o médio baixo e/ou baixo padrão socioeconômico.

\section{DISCUSSÃO}

Florianópolis/SC é uma capital brasileira, localizada na Região Sul do Brasil, que sofreu um acelerado processo de transformação social, político, cultural e econômico a partir da década de 80 , desencadeando alteração do perfil populacional a partir de movimentos migratórios internos e externos.

O planejamento e execução de ações preventivas para DST/AIDS em Florianópolis/SC encontra diversas dificuldades, seja pelo crescimento populacional, como por suas características. Neste sentido, um diagnóstico epidemiológico que analise a vulnerabilidade potencial das pessoas ao HIV/AIDS, bem como um diagnóstico socioeconômico ocupacional destes subgrupos, pode colaborar para o delineamento de uma política de prevenção que atinja toda a população, bem como os subgrupos mais vulneráveis.

A variação das atividades exercidas na Categoria Não - Manual, destaca que as pessoas que vivem com HIV/AIDS em Florianópolis/SC, têm como ocupações atuar como ‘Outros Profissionais Liberais' e ‘Empresários por Conta Própria'. A redução de percentual nas duas décadas do estudo faz inferir que indivíduos com melhor qualidade de vida tendem a se proteger para o HIV/AIDS.
Na Categoria Manual, a ocupação 'Serviços Pessoais' e 'Serviços Gerais' prevalece entre as pessoas que vivem com HIV/AIDS em Florianópolis/SC. A atividade na Indústria Tradicional se destaca entre os homens que vivem com HIV/AIDS em Florianópolis/SC.

O estudo apresenta diferenças significativas em relação às atividades laborais exercidas pelas pessoas que vivem com AIDS em Florianópolis/SC. Na Categoria Não Ocupada, o resultado aponta a vulnerabilidade de mulheres que desenvolvem tarefas como domésticas, babás e no lar. Muitas mulheres tendem a desenvolver atividades para geração de renda na informalidade, a fim de protegerem-se da exposição de seu diagnóstico, do julgamento, preconceito e estigma que ainda é gerado por esta condição.

Na Categoria Não Ocupado, o resultado apresenta a vulnerabilidade potencial do homem na extremidade da pirâmide etária (jovem e idoso), que exerce ocupação como Estudante, Aposentado/Pensionista.

Em Florianópolis/SC, em duas décadas de estudo da AIDS, o retrato da ocupação laboral e o status socioeconômico ocupacional das pessoas que vivem com HIV/AIDS, aponta subgrupos com menor qualidade de vida e os vulneráveis potenciais, assim como a tendência de reprodução de comportamento dos seus pares pessoais e/ou sexuais.

Observa-se entre as mulheres que vivem com HIV/AIDS em Florianópolis/SC, que desenvolvem ocupação como 'Afazeres Domésticos', o predomínio das mulheres casadas e de menor escolaridade. As mulheres infectadas e doentes pelo HIV/AIDS tendem a ocultar sua condição, mantendo o trabalho em casa, na lida dos lares, dos maridos e dos filhos; e fora do lar exercendo atividades em sua grande maioria como camareiras, garçonetes, vendedoras, coletoras de materias reciclados, entre outras atividades. Os maiores coeficientes de incidência neste subgrupo sinaliza sua vulnerabilidade social, com adoecimento pelo HIV/AIDS em decorrência de aspectos individuais e coletivos ${ }^{(10-13)}$.

Estudo realizado com mulheres brasileiras que exercem Atividades Domésticas, observou-se acréscimo de $20 \%$ por ano de mulheres que vivem com HIV/AIDS, enquanto entre outras ocupações, ocorre redução de $20 \%$ entre as mulheres que vivem com HIV/AIDS(10). Um aspecto que tende a explicar a elevação de mulheres que vivem com HIV/AIDS nas duas décadas de estudo, está 
relacionado à realização do teste para HIV no pré-natal e o teste rápido realizado nas maternidades na hora do parto. Estudo com mulheres, realizado pelo Ministério da Saúde em 2003, identificou que $28 \%$ das mulheres com idade de 14 e mais anos, realiza o exame HIV pelo menos uma vez na vida. No entanto, na classe social $D$ e $E$, observou-se o menor percentual na realização do exame, com cobertura do teste anti-HIV na gestação de 57,4\% no ano de 2003, o que sinaliza a precarização do acesso destes segmentos sociais ao serviço de saúde ${ }^{(12)}$.

A ocupação do indivíduo apresenta relação com sua escolaridade, e no início da epidemia da AIDS em Florianópolis/SC, observa-se maior proporção de pessoas infectadas nos ocupantes do Status Socioeconômico Ocupacional denominado alto $\left(1^{\circ} \mathrm{ESO}\right)$ e médio alto $\left(2^{\circ}\right.$ ESO), deste modo, com mais anos de estudo e melhores ocupações laborais. Com o passar dos anos, a AIDS passa a incrementar suas estatísticas com indivíduos com poucos anos de estudo e Status Socioeconômico Ocupacional denominado médio baixo ( $4^{\circ} \mathrm{ESO}$ ) e baixo ( $\left.5^{\circ} \mathrm{ESO}\right)$, que compõem grupos menos favorecidos e com menor escolaridade. Infere-se que a inacessibilidade de informações sobre a AIDS, a dificuldade de acessar os serviços de saúde e a posição socioeconômica desfavorável na hierarquia social, compromete a acessibilidade aos direitos de saúde do cidadão(3-4,11).

No $4^{\circ}$ ESO, homens que vivem com HIV/AIDS, tendem a exercer atividades como pedreiros, vigias, garçons, entre outros, contribuindo menos para a previdência social e apresentando alta rotatividade de trabalho. No $5^{\circ}$ ESO, as pessoas que vivem com HIV/AIDS exercem atividades como autônomos, lavadeiras, serventes de pedreiro, entre outros. Apresentam pouco tempo de trabalho na profissão, alta jornada de trabalho e poucos contribuem para a previdência social. Conforme decresce o ESO, decresce o nível de escolaridade, de rendimento, de manutenção do estado de saúde, da qualidade de vida, aumentando consequentemente o risco de exposição às DST/AIDS ${ }^{(9,14-}$ 16).

A educação formal é o diferencial na mobilidade social e acesso a bens de consumo, como os serviços de saúde, insumos de prevenção e materiais sobre promoção da saúde e prevenção das DST/AIDS. A pouca e/ou nenhuma escolaridade, acarreta as pessoas que vivem com HIV/AIDS, perda em termos de renda e desigualdade socioeconômica ocupacional, associada ainda, ao estigma e preconceito. A cultura de uma visão educacional baixa entre mulheres dos $4^{\circ}$ e $5^{\circ}$ ESO, resulta em orientações à saúde e aconselhamento incompletos e com baixa aderência(10,15). Dessa forma, essas mulheres pouco se beneficiam com orientações sobre prevenção às DST/AIDS, com pouco resultado na diminuição da vulnerabilidade potencial feminina ${ }^{(4,11-13)}$.

O diagnóstico de AIDS é impactante em todas as categorias socioeconômicas, entretanto para pessoas que vivem com HIV/AIDS dos $4^{\circ}$ e $5^{\circ} \mathrm{ESO}$, pode resultar até mesmo na perda do emprego e dificuldade de inserção/reinserção no mercado de trabalho(11).

As pessoas que vivem com HIV/AIDS afastam-se do trabalho formal, exercendo atividades laborais informais, de baixa escolaridade, que thes proporcione uma renda básica. O processo leva a pauperização desses segmentos populacionais, resultando em perda gradual das condições de sobrevivência, falta de adesão ao tratamento, aumento do sofrimento pessoal, depressão e ao isolamento social(16-18).

\section{CONCLUSÃO}

A análise da situação ocupacional e socioeconômica ocupacional das pessoas que vivem com HIV/AIDS residentes em Florianópolis/SC, sinaliza a dinâmica socioeconômica dos subgrupos vulneráveis e os vulneráveis potenciais ao HIV/AIDS, como jovens, idosos, mulheres que se dedicam aos afazeres domésticos, pessoas de baixa e/ou nenhuma escolaridade e baixa condição socioeconômica. Faz-se importante ressaltar esta problemática em uma cidade como Florianópolis-SC, que apesar de reconhecida por sua qualidade de vida, não escapa dos problemas sociais existentes nas grandes cidades, e que em muito repercutem na saúde das pessoas.

Ratifica-se a importância de realizar o diagnóstico epidemiológico e socioeconômico ocupacional das pessoas que vivem com HIV/AIDS, a fim de planejar políticas que possam responder à dinâmica da epidemia. Sugere-se a disponibilização parcial de dados do Sistema de Agravo de Notificação Nacional (SINAN-AIDS) e Sistema de Informação de Mortalidade (SIM) de informação como o SINAN, aos profissionais de saúde, com intuito de conhecer a distribuição global dos casos de AIDS em seu território de atuação e suas tendências. 
Os resultados deste estudo trazem como desafio para instituições e profissionais de saúde, o planejamento de políticas que favoreçam a acessibilidade aos serviços de saúde aos subgrupos mais vulneráveis à epidemia, ou seja, aqueles mais desfavorecidos socioeconomicamente. Esta acessibilidade no entanto, se repercute também em ações de prevenção e assistência que sejam condizentes

\section{REFERÊNCIAS}

1. Ministério da Saúde. AIDS no Brasil epidemia estabilizada e concentrada em populações de maior vulnerabilidade. [Internet]. 2011. 15 p. [cited 2012 Fev 28]. Available from:

http://www.aids.gov.br/sites/default/files/anexos/publicacao/2011/ 50652/resumo_anal tico_dos dados do boletim_epidemiol 9282 4.pdf

2. Ministério da Saúde. Boletim epidemiológico DST/AIDS. [Internet]. 2011. 26 p. [cited 2012 Fev 28]. Available from: http://www.aids.gov.br/sites/default/files/anexos/publicacao/2011/ 50652/boletim aids 2011 preliminar3 pdf 20265.pdf 3. Programa das Nações Unidas para o Desenvolvimento. Desenvolvimento humano e IDH. [Internet]. 2012. [cited $2012 \mathrm{Fev}$ 20]. Available from: http://www.pnud.org.br/idh/

4. Instituto Brasileiro de geografia e Estatística. Educação e trabalho. [Internet]. [cited 2012 fev 22]. Available from:

http://www.ibge.gov.br/home/estatistica/populacao/condicaodevid a/indicadoresminimos/suppme/analiseresultados2.shtm

5. Costa-Couto MH. A vulnerabilidade da vida com HIV/AIDS. [tese]. Doutorado em Politíca, Planejamento e Administração em Saúde. Rio de janeiro: Universidade Estadual do Rio de Janeiro. 2007. 6. Barbosa Junior A, Pascom ARP, Szwarcwald CL, Dhalia CBC, Monteiro L, Simão MB. Indicadores propostos pela UNGASS e o monitoramento da epidemia da AIDS no Brasil. Rev Saúde Pública 2006;40(Supl):94-100.

7. Fonseca MG, Travassos C, Bastos FI, Silva NV, Szwarvwald CL. Distribuição social da AIDS no Brasil, segundo participação no mercado de trabalho, ocupação e status sócio-econômico dos casos de 1987 a 1998. Cad. Saúde Pública. 2003; 19(5): 1351-63. 8. Secretaria Estadual da Saúde de Santa Catarina. Diretoria de Vigilância Epidemiológica. Carnaval 2011. [Internet]. 2011. [cited 2012 Fev 22]. Avaible from:

http://www.dive.sc.gov.br/conteudos/gerencia dst aids/noticias/20 11/Carnaval_2011.pd

9. Fonseca MG, Szwarcwald CL, Bastos FI. A sociodemographic analysis of the AIDS epidemic in Brazil, 1989-1997. Rev Saúde Pública. 2002; 36(6):678-85.

10. Januzzi PM. Estratificação socioocupacional para estudos de mercado e pesquisa social no brasil. São Paulo Em Perspectiva. 2003; 17(3-4):247-54.

11. Braga PE, Cardoso MRA, Segurado AC. Gender differences among persons with HIV admitted to reference center in São Paulo, Brazil. Cad. Saúde Pública. 2007; 23(11): 2653-62.

12. Dourado I, Veras MASM, Barreira D, Brito AM. AIDS epidemic trends after the introduction of antiretroviral therapy in Brazil. Rev Saúde Pública. 2006;40(Supl):9-

13. Padoin SMM, Souza IEL. A ocupação da mulher com HIV/AIDS: o cotidiano diante da (im)possibilidade de amamentar. DST j. bras. doenças sex. transm. 2006;18(4):241-46.

14. Santos W, Munari DB, Medeiros M. O grupo de mulheres que vivem e convivem com HIV/AIDS: um relato de experiência. Rev. Eletrônica Enfer. [Internet]. 2009 [cited 2010 Ago 25];11(4):1043-8. Available from:

http://www.fen.ufg.br/revista/v11/n4/v11n4a32.htm

15. Kourrouski MFC,Lima RAG. Treatment adherence: the experience of adolescents with HIV/AIDS. Rev. Latino-Am. Enfermagem.

2009;17(6):947-52. com o universo social, econômico, educacional e cultural destes grupos, no sentido de produzir intervenções efetivas na vida destas pessoas. Outrossim, destaca-se a importância de compartilhar experiências exitosas no controle e diminuição dos casos de HIV/AIDS no Brasil, tendo em vista a mudança no perfil dos vulneráveis potenciais.

16. Maliska ICA, Padilha MICS. AIDS: a experiência da doença e a construção do itinerário terapêutico. Rev. Eletr. Enf. [Internet]. 2007. [cited 2012 Mar 06];9(3): 687-699. Available from: URL: http://www.fen.ufg.br/revista/v9/n3/v9n3a09.htm

17. Fonseca MG, Bastos FI, Derrico M, Andrade CL, Travassos C, Szwarcwald CL. Aids and level of education in Brazil: temporal evolution from 1986 to 1996. Cad Saúde Pública. 2000;16(Sup 1):7787.

18. Ferreira RCM, Figueiredo MA. Reinserção no mercado de trabalho. Barreiras e silêncio no enfrentamento da exclusão por pessoas com HIV/AIDS. Medicina. 2006;39(4):591-600.

19. Almeida ANS, Silveira LC, Silva MRF, Araújo MAM e Guimarães TA. Subjectivity and sexuality production in women living with HIV/Aids: a Sociopoetic Production. Rev. Latino-Am. Enfermagem. 2010;18(2):163-69.

Artigo recebido em 25/08/2011.

Aprovado para publicação em 31/01/12.

Artigo publicado em 30/09/2012. 